\section{CPICH power optimisation by means of simulated annealing in an UTRA-FDD environment}

\author{
M. Garcia-Lozano, S. Ruiz and J. Olmos
}

In the framework of an UTRA-FDD system, simulated annealing is used to optimise the values of powers assigned to common pilot channel (CPICH) signals in order to force mobiles to transmit to the best option cell. Results show the existence of optimal configurations that decrease the cells load. Consequently, capacity can be increased.

Introduction: Because of the use of rake receivers and the introduction of a soft handover scheme in UTRA-FDD, there are areas of operation in which user equipments (UE) are connected to a group of base stations (BS) known as the 'active set' (AS). Since the network orders the UE to add a BS to its AS according to quality measurements made on the primary scrambling code of the common pilot channel (CPICH or pilot) [1], it may be inferred that the traffic can be balanced among the cells by means of $\mathrm{CPICH}$ power variations. Moreover, in WCDMA environments, interference level has a great impact in the power a UE has to transmit. Consequently, an intelligent choice in the pilot powers could lead the system into optimal or quasioptimal configurations in which the uplink (UL) load is minimised and a higher capacity is achieved.

One way to tackle the problem has been suggested from an allocation point of view; that is a set of possible $\mathrm{CPICH}$ powers has to be assigned to BSs so that a cost function is minimised. As the aim is that the UL load factor $\eta_{U L}(1)$ of all BSs should be jointly reduced, it seems clear that the cost function should be related to $N_{T}(m)$. Note that $N_{T}(m)$ is the total received power (signal, interference and noise) and $N(m)$ is the thermal noise, both measured at BS ' $m$ ':

$$
\eta_{U L}(m)=\frac{N_{T}(m)-N(m)}{N_{T}(m)}
$$

Consequently, (2) was eventually adopted as a good option. Not only by its performance but also because its calculus was not time-consuming. Note that $P_{T X}(i)$ is the transmission power of the $i$ th UE and $I$ is the number of UEs in the system:

$$
\operatorname{cost}=\sum_{i=1}^{I} P_{T X}(i)
$$

Since the objective is not to obtain absolute CPICH values for a specific scenario but to investigate a technique, only conversational services have been considered. Once asymmetric data services are included, the cost function will have to take into account downlink (DL) considerations.

There are also some constraints that must be taken into account by the solution: 1, Users not achieving the SIR target in a certain cell must be $<5 \%$. 2, The range of possible $\mathrm{CPICH}$ powers is established according to two restrictions that should be defined in a previous phase of the planning process: $(a)$ the minimum eligible power must guarantee a desirable coverage; and $(b)$ the maximum eligible power must avoid illogical situations, in which DL capacity could be jeopardised. The power step choice will define the range of possible pilot levels. An excessively low step implies a wide range of solutions with the same cost and thus longer simulations. On the other hand, a big step could produce a loss of important quasi-optimal solutions.

Application of simulated annealing: Mathematically speaking, the problem has been posed as a combinatorial NP-complete one. Several heuristic algorithms have been developed to tackle it. The proposed technique is based on simulated annealing (SA), which is one of the most popular because of its simplicity and the asymptotic convergence on optimal solutions [2]. It consists of a random search algorithm that efficiently explores the solutions space (Table 1).

Initially, starting with a random solution accomplishing the constraints, the temperature is gradually increased until the ratio ' $r$ ' (number of accepted solutions/number of proposed solutions) reaches $85 \%$. The solution and temperature at that point are considered as the initial ones. Thus independence between the initial solution and the final one are guaranteed. On the other hand, the algorithm will be considered to have reached convergence when the ratio ' $r$ ' is under $1 \%$.
Table 1: Simulated annealing

\begin{tabular}{|c|c|}
\hline 1 & Obtain initial solution $S$ and temperature $T$ \\
\hline 2 & $C \leftarrow \operatorname{cost}$ of $S$ \\
\hline 3 & Generate new solution $S$ \\
\hline 4 & $C \leftarrow$ cost of $S$ \\
\hline \multirow{2}{*}{5} & Accept $S$ as the current solution $S$ with probability $p:$ \\
\cline { 2 - 3 } & $p=\exp [(C-C) / T] \quad$ if $C>C$ \\
\cline { 2 - 3 } 6 & $p=1 \quad$ if $\quad C<C$ \\
\hline 7 & If equilibrium condition has not been reached, go to 3 \\
\hline 8 & If termination criterion has not been reached, go to 3 \\
\hline
\end{tabular}

The generation of a new solution consists of a perturbation over the current one: one random pilot power accomplishing the constraints and one random BS in the system are chosen. Next the Metropolis criterion is evaluated: movements towards worse solutions are allowed with a certain probability depending on the system temperature; consequently the algorithm does not get trapped in local minima. The quality of the proposed solution will depend on the cooling strategy, the termination criterion and the equilibrium condition. Actually, a tradeoff between the speed for the convergence and the quality of the solution arises. In our work, the temperature has been updated according to (3) because, unlike typical geometric cooling, it has been shown to preserve the convergence theory towards the global minimum as much as possible [2]:

$$
T_{\text {new }}=T_{\text {old }}\left(\frac{1+T_{\text {old }} \ln (1+\delta)}{3 \sigma}\right)^{-1}
$$

where $\delta$ is an adjustable parameter that allows controlling the cooling speed and $\sigma$ is the standard deviation of the accepted solutions cost values during the period of time in which the temperature was $T_{\text {old }}$.

Simulations and results: All the simulations have been done by means of a sequence of uncorrelated snapshots. A fast macroscopic algorithm [3] has been implemented to solve the power control equations. However, since a UE in soft handover is treated as several different radiolinks, the macroscopic resolution is followed by a fine adjust [4]. The algorithm jumps automatically to a classical iterative algorithm only when there is no macroscopic solution.

The simulation platform is based on that described in [5]. Therefore we are working within a realistic scenario with 13 cells based on real attenuation measurements from a GSM network. The minimum eligible power could have been independently established for each cell. However, for the sake of simplicity in our simulations the BSs will share the same value: $27 \mathrm{dBm}$. The maximum level was set to $33 \mathrm{dBm}$ and the power step to $0.1 \mathrm{~dB}$. Regarding the AS configuration, a maximum of $3 \mathrm{BSs}$ and a macrodiversity window of $3 \mathrm{~dB}$ was shown to be a good tradeoff between the UL and the DL performance in [5].

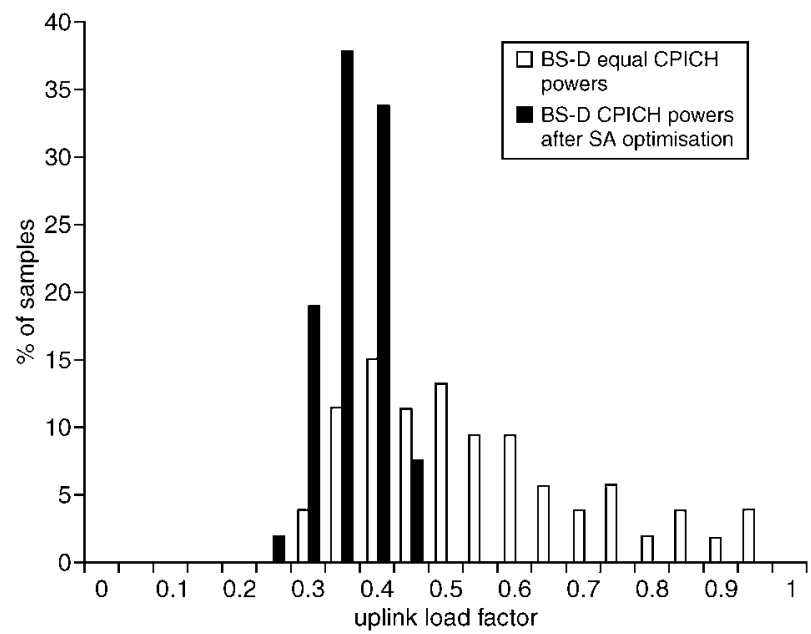

Fig. 1 Uplink load factor in cell ' $D$ ' before and after applying SA

Fig. 1 shows a histogram of the UL load factor for a random cell in the system before (equal pilot powers) and after the optimisation 
process; a large reduction achieved in the load with the proposed technique can be seen. The algorithm is able to find combinations of pilot powers such that the UL interference is minimised and, consequently, capacity can be increased. Not only the mean obtained after averaging different snapshots is lower, but also the standard deviation has been reduced too. Both reductions were obtained in $100 \%$ of the BSs, as can be seen in Fig. 2 for the mean case.

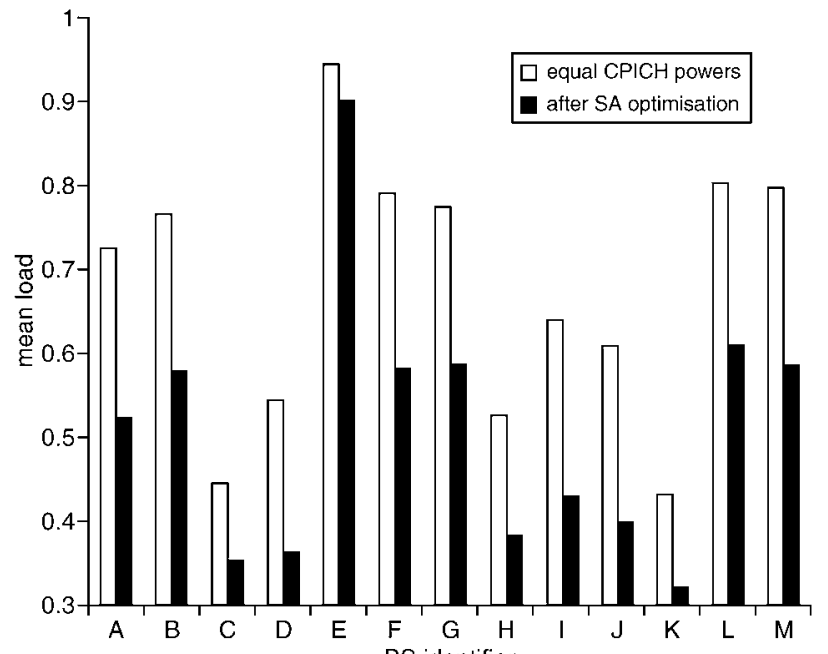

BS identifier

Fig. 2 Mean load in each cell before and after applying $S A$

Conclusions: A technique based on simulated annealing has been presented in order to decide a combination of pilot powers so that load factors of the cells in a system are reduced. The operation of the developed algorithm was shown to be very good since it achieved reduction in $100 \%$ of the cells. As a consequence this reveals that, after applying it, operators could increase system capacity. On the basis that traffic patterns are repetitive during a day and in different days of the week, SA could be combined with techniques of estimation and prediction of traffic. This would imply a training of the system for those particularly differentiated periods of time so that pilot powers could change automatically.

(C) IEE 2003

Electronics Letters Online No: 20031067

14 July 2003 DOI: 10.1049/el:20031067

M. Garcia-Lozano, S. Ruiz and J. Olmos (Department of Signal Theory and Communications, Polytechnic University of Catalonia (UPC), C/ Jordi Girona 1-3, Módulo D4, 08034, Barcelona, Spain)

E-mail: mariogarcia@tsc.upc.es

\section{References}

1 3GPP (TS 25.133 v5.4.0 2002-10)

2 AARTS, E.H.I., and KORST, J.H.H.: 'Simulated annealing and Boltzmann machines: a stochastic approach to combinatorial optimization and neural computing' (Wiley Publishers, New York, USA, 1989)

3 MENDO, L., and HERNANDO, J.M.: 'On dimension reduction for the power control problem', IEEE Trans. Commun., 2001, 49, (2), pp. 243-248

4 RUIZ, S., GARCIA-LOZANO, M., and OLMOS, J.: 'Admission and cell congestion analysis through dynamic power allocation'. PIMRC 2002, 13th IEEE International Symposium on personal, indoor and mobile cadio communications, Lisbon, Portugal, 2002, Vol. 1, pp. 11-15

5 GARCIA-LOZANO, M., et al.: 'Analysing UTRA-FDD pilot power and active set configuration in a real urban scenario'. PIMRC 2003. 14th International Symposium on personal, indoor and mobile radio communications, Beijing, China, 2003 (accepted paper) 